\title{
Protein expression profiles that underpin the preventive and therapeutic potential of Moringa oleifera Lam against azoxymethane and dextran sodium sulfate-induced mouse colon carcinogenesis
}

\author{
PHATCHARIYA PHANNASIL ${ }^{1}$, SITTIRUK ROYTRAKUL $^{2}$, NARUMON PHAONAKROP ${ }^{2}$, \\ PIENGCHAI KUPRADINUN ${ }^{3}$, SIRINTIP BUDDA ${ }^{4}$, CHANIPHUN BUTRYEE ${ }^{4}$, \\ CHAREEPORN AKEKAWATCHAI $^{5^{*}}$ and SIRIPORN TUNTIPOPIPAT ${ }^{4 *}$
}

\author{
${ }^{1}$ Thalassemia Research Center, Institute of Molecular Biosciences, Mahidol University, Nakhon Pathom 73170; \\ ${ }^{2}$ Funtional Ingredients and Food Innovation Research Group, National Center for Genetic Engineering and Biotechnology, \\ National Science and Technology Development Agency, Pathumthani $12120 ;{ }^{3}$ Section of Animal Laboratory, \\ Research Division, National Cancer Institute, Bangkok 10400; ${ }^{4}$ Food Cluster, Institute of Nutrition, \\ Mahidol University, Nakhon Pathom 73170; ${ }^{5}$ Department of Medical Technology, Faculty of \\ Allied Health Sciences, Thammasat University, Pathumthani 12121, Thailand
}

Received October 5, 2019; Accepted April 1, 2020

DOI: $10.3892 / \mathrm{ol} .2020 .11730$

\begin{abstract}
Previous studies in a mouse model have indicated the anticancer potential of boiled Moringa oleifera pod (bMO)-supplemented diets; however, its molecular mechanisms are still unclear. Therefore, the present study aimed to explore the protein expression profiles responsible for the suppressive effect of bMO supplementation on azoxymethane $(\mathrm{AOM}) /$ dextran sodium sulfate (DSS)-induced mouse colon carcinogenesis. Analysis by gel electrophoresis and liquid chromatography-tandem mass spectrophotometry demonstrated that there were 125 proteins that were differentially expressed in mouse colon tissues between 14 experimental
\end{abstract}

Correspondence to: Dr Chareeporn Akekawatchai, Department of Medical Technology, Faculty of Allied Health Sciences, Thammasat University, 99 Moo 18, Piyachart Bldg, Paholyotin Road, Pathumthani 12121, Thailand

E-mail: cakekawatchai@gmail.com

Dr Siriporn Tuntipopipat, Food Cluster, Institute of Nutrition, Mahidol University, Putthamonthon 4 Road, Putthamonthon, Nakhon Pathom 73170, Thailand

E-mail: siriporn.tun@mahidol.ac.th

${ }^{*}$ Contributed equally

Abbreviations: AOM, azoxymethane; CACC, colitis-associated colon cancer; DSS, dextran sodium sulfate; MO, Moringa oleifera; GeLC-MS/MS, gel electrophoresis and liquid chromatography-tandem mass spectrometry

Key words: AOM/dextran-induced mouse colon carcinogenesis, $\mathrm{MO}$, proteomics, GeLC-MS/MS groups of mice. The differentially expressed proteins are involved in various biological processes, such as signal transduction, metabolism, transcription and translation. Venn diagram analysis of the differentially expressed proteins was performed in six selected mouse groups, including negative control, positive control mice induced by AOM/DSS, the AOM/DSS groups receiving preventive or therapeutic bMO diets and their bMO-supplemented control groups. This analysis identified 7 proteins; $60 \mathrm{~S}$ acidic ribosomal protein $\mathrm{P} 1$ (Rplp1), fragile X mental retardation, cystatin 9, round spermatids protein, zinc finger protein 638 , protein phosphatase 2C (Ppm1g) and unnamed protein product as being potentially associated with the preventive and therapeutic effects of bMO in AOM/DSS-induced mouse colon cancer. Analysis based on the search tool for interactions of chemicals (STITCH) database predicted that Rplp1 interacted with the apoptotic and inflammatory pathways, whereas Ppm1g was associated only with inflammatory networks. This proteomic analysis revealed candidate proteins that are responsible for the effects of bMO supplementation, potentially by regulating apoptotic and inflammatory signaling networks in colorectal cancer prevention and therapy.

\section{Introduction}

Colorectal cancer (CRC) is the third most common cancer and the fourth highest for cancer-associated deaths, accounting for 700,000 deaths worldwide in 2012 (1). Incidence rates of $\mathrm{CRC}$ are increasingly reported in industrialized countries with a moderate and high Human Development Index (2). There is no effective early detection method for CRC and most of the cases are diagnosed at advanced stages of the disease. Conventional radiotherapy and chemotherapy regimens for CRC have a low cure rate and cause adverse side effects. CRC 
incidence could be potentially preventable by the consumption of a healthy diet (3). Therefore, diet modification and education on healthy lifestyle are necessary to reduce the incident rate of CRC. CRC risk is inversely associated with the total intake of fruit and vegetables in the diet, which are excellent sources of phytochemicals (4). Several in vitro and in vivo studies have demonstrated that phytochemicals, such as sulforaphane, diferuloylmethane and resveratrol, serve antioxidant, anti-inflammatory and anticancer roles by modulating specific signaling pathways including $\mathrm{Bax} / \mathrm{Bcl}-2$-dependent apoptosis and p53- and p21-mediated cell cycle arrest, to inhibit the progression and promotion of CRC (3).

Moringa oleifera Lam. (MO) belongs to the Moringaceae family. Various parts of this vegetable, including leaves and pods with seeds, are commonly consumed as part of the regular diet in different parts of the world. MO has a wide range of health benefits, including anti-inflammatory, anticancer, hepatoprotective and neuroprotective functions (5). It has been reported that $\mathrm{MO}$ has the medicinal potential including anti-bacterial, anti-inflammatory, anti-oxidant and antitumor roles (6). Due to these reported functions, the understanding of the pharmacological functions and underlying mechanisms of MO action need to be further explored. Bioactive compounds have been isolated from MO leaves, pods and seeds, such as vitamins, carotenoids, polyphenol, phenolic acids, flavonoids, alkaloids, niazirin, niazimicin, niazin A, benzyl-isothiocyanate, benzyl-thiocarbamate and glucomoringin (7-9). Extracts from MO leaves and bark have efficacy as anticancer agents as demonstrated by them inducing apoptosis in a HCT- 8 colorectal cancer cell line (10). The essential oil from MO seeds and MO dichloromethane extract had significant cytotoxic, anti-oxidant and anti-proliferative effects in the Caco-2 colorectal cell line $(11,12)$. Recent studies have also indicated that MO lam extracts have cytotoxic and apoptotic effects on human lymphoid and monocytoid cell lines potentially due to the cross-kingdom regulation by miRNA present in MO $(13,14)$.

Although various parts of MO exhibit biological functions, MO pods have drawn considerable attention (15-17) as they are common ingredients in traditional Asian soups from Thailand, India and Philippines. Boiled MO (bMO) pods exhibited strong anti-inflammatory effects in LPS-induced murine macrophages (15). The chemopreventive and therapeutic potential of bMO pods were demonstrated in azoxymethane $(\mathrm{AOM}) /$ dextran sodium sulfate (DSS)-induced mouse colon cancer. The results indicated that tumor formation and multiple tumors were significantly decreased in the AOM/DSS-induced mice receiving bMO compared with AOM/DSS-induced mice $(16,17)$. The therapeutic efficacy of boiled MO was partially due to its ability to induce apoptotic cell death in colon tissues, by upregulating the pro-apoptotic protein Bax and decreasing the anti-apoptotic protein Bcl-2 expression (17). Preventive treatment with bMO in mice reduced the expression of inflammatory mediators, inducible nitric oxide synthase (iNOS) and cyclooxygenase 2 (COX-2) (16). However, the underlying protective and therapeutic mechanisms of bMO in exerting anticancer effects remains unclear and requires further elucidation.

The present study aimed to investigate the molecular mechanisms for the suppressive effect of bMO supplementation on azoxymethane (AOM)/dextran sodium sulfate (DSS)-induced mouse colon carcinogenesis. The proteomic analysis of colon tissues from AOM/DSS-induced mice receiving preventive and therapeutic bMO diets was performed, in order to explore the protein expression profiles in response to bMO consumption. A group of candidate proteins were identified by Venn diagram analysis and their protein-protein interaction network and association with apoptotic and inflammatory signaling pathways were predicted based on the STITCH database. These data provide an insight into the molecular mechanisms that underpin the suppressive effects of bMO in a CRC mouse model.

\section{Materials and methods}

Chemicals. AOM was purchased from Merck KGaA and dextran sodium sulfate (molecular weight, 36,000-50,000) was purchased from MP Biomedicals, LLC. For animal diet preparation, AIN-76 mineral and vitamin mixtures were purchased from MP Biomedicals, LLC and CLEA Japan, Inc. Vitamin K1 was kindly provided by DSM Nutritional Products Ltd. and cellulose (SOLKA-FLOC ${ }^{\circledR} 200$ FCC) from FS\&D Corp. Sodium caseinate was the product of Erie Foods International, Inc.

Animal diet preparation. MO boiled pods were prepared as previously described (16). In brief, MO pods were boiled in boiling water at ratio 1:1 for $7 \mathrm{~min}$ and left them cool down at $25^{\circ} \mathrm{C}$. The boiled MO and water were blended, grinded in an electronic grinder and freeze-dried by lyophilization at $-30^{\circ} \mathrm{C}$ and termed as bMO. The bMO was packed in vacuum bags and stored at $-20^{\circ} \mathrm{C}$ until use. The experimental diets were prepared by mixing bMO at concentrations of 1.5, 3.0 and $6.0 \%$ with the basal AIN-76 diet (Table SI). Animal diet preparation was prepared according to the protocol previously described $(16,17)$. The bMO content in animal diets was equivalent to 10, 20 and 40 times of human consumption (Food consumption survey data of Thai population, 2006) (18).

Animals and experimental design. A total of 112 male ICR mice [age, 3 weeks old and weight, $15 \pm 3 \mathrm{~g}$ (mean $\pm \mathrm{SD}$ )] were maintained at the Laboratory Animal Facility of the National Cancer Institute according to the Institute Care Guidelines as previously described $(16,17)$. The study protocol was approved by the Animal Ethics Committees of the National Cancer Institute and Mahidol University (Thailand). Mice were acclimatized and fed with the modified AIN-76 semi-purified diet (basal diet), described in Table SI, and had access to water ad libitum for 5 days. After acclimatization, mice were randomly divided into 14 groups ( $n=8 /$ group) and fed with basal AIN-76 or AIN-76 diet containing bMO. The experimental design is presented in Fig. 1. In brief, group 1, negative control was fed the basal diet throughout the 20 -week experimental period. Group 2, positive control for colitis-associated colon cancer (CACC), was intraperitoneally injected with AOM $[10 \mathrm{mg} / \mathrm{kg}$ body weight (BW)] at the $3 \mathrm{rd}$ week, and this was followed by administration of $2 \%$ DSS in drinking water at the 4 th week for one week. The mice in Group 2 were fed with basal diets for the 20-week experimental period. Group 3-5 mice were fed with basal diet supplemented with bMO at 1.5, 


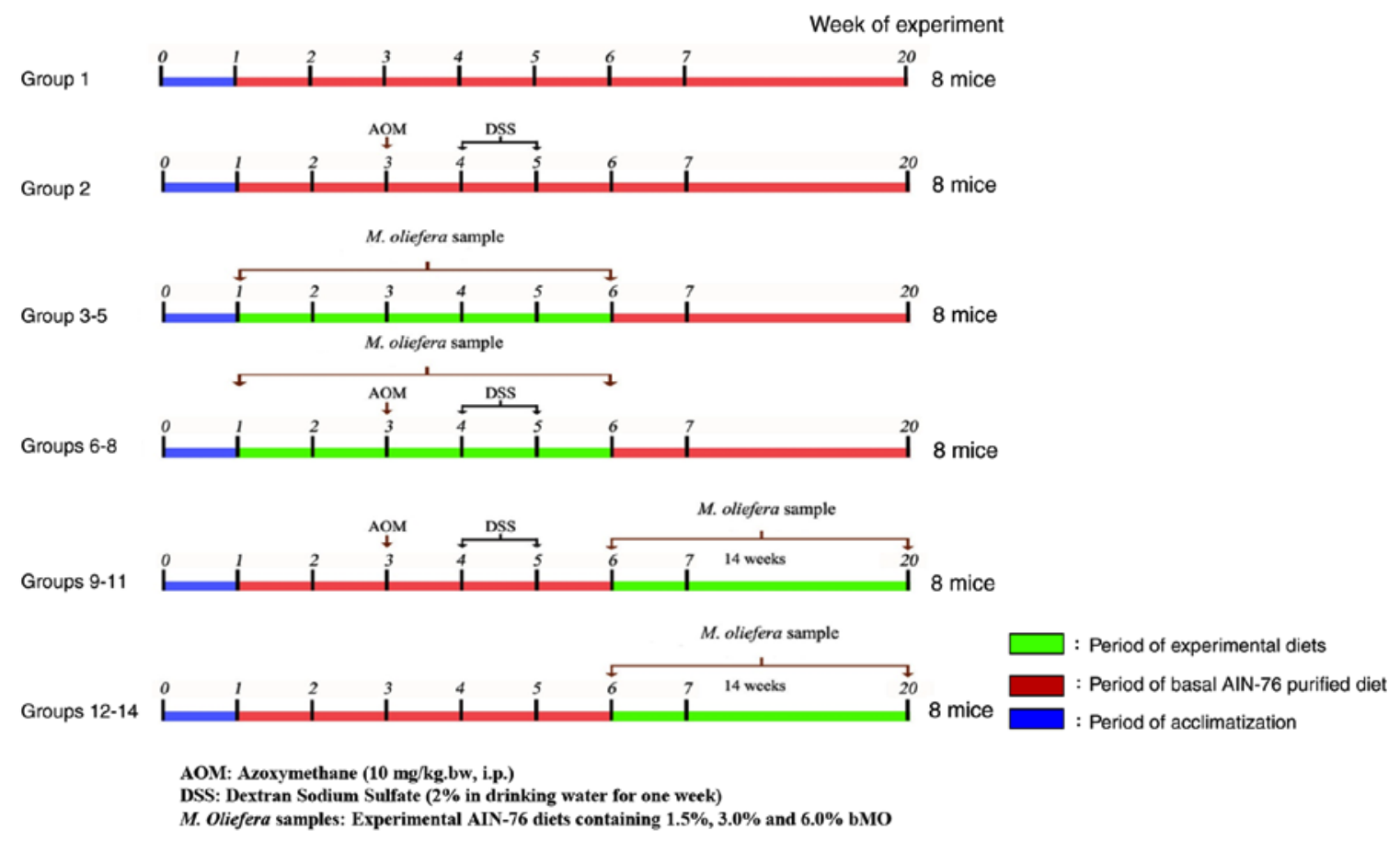

Figure 1. Experimental design for studying effects of bMO against mouse colon carcinogenesis. AOM, azoxymethane; DSS, dextran sodium sulfate; bMO, boiled Moringa oleifera pod; M., Moringa; AIN-76, American Institute of Nutrition-76 (16,17).

3.0 and $6.0 \%$, respectively for 5 weeks (from week 1 to 5) and then continued with the basal diet until the end of experiment. Group 6-8 mice, designated to evaluate the preventive effect of $\mathrm{bMO}$ in CACC mice, were fed with basal diet containing bMO at $1.5,3.0$ and $6.0 \%$, respectively for 2 weeks prior to giving $\mathrm{AOM}$ at week 3 of experimentation; this was followed by administration with 2\% DSS in drinking water in the 4th week for 1 week. Group 6-8 mice were fed with experimental diets for 5 weeks, followed by basal diet until the end of the experiment. Group 9-11 mice, designated to assess the therapeutic effect of bMO in CACC mice, were intraperitoneally injected with AOM (10 mg/kg BW) at week 3, followed by the administration of $2 \%$ DSS in drinking water at week 4 for a week. Mice were then fed with the basal diets containing 1.5, 3.0 and $6.0 \%$ bMO, respectively at week 6 until the end of the experiment. Group 12-14 mice were fed with basal diet until week 6 , followed by experimental diets containing 1.5, 3.0 and 6.0\% bMO, respectively, until the end of the experiment. At the end of the study, mice were euthanized by replacing $30 \%$ of the chamber volume per minute with carbon dioxide. Colons were excised and examined for the presence of tumors. As described previously, colons from group 1, 2 and 3-8 mice were examined for the preventive effect of bMO (16), and those from group 1, 2 and 9-14 mice were examined for the therapeutic effect (17) on the AOM/DSS-induced carcinogenesis. The data on tumor nodule incidence in 14 groups of mice described in the previous reports are summarized in Table I. The portion of colons was frozen until use for the present proteomic analysis.

Tissue protein extraction. Frozen colon tissue was washed with phosphate buffer saline (PBS; pH 7.4) and centrifuged $\left(3,000 \times \mathrm{g}, 5 \mathrm{~min}, 4^{\circ} \mathrm{C}\right)$. Ice-cold RIPA buffer [50 mM Tris- $\mathrm{HCl}$ pH 7.4, $150 \mathrm{mM} \mathrm{NaCl}, 1 \mathrm{mM}$ EDTA, $0.25 \%$ sodium deoxycholate, $1 \%(\mathrm{v} / \mathrm{v}) \mathrm{NP}-40,1 \mathrm{mM}$ DTT and $1 \mathrm{X}$ protease inhibitor cocktail (Roche Diagnostics) was added to the tissue and was placed on ice for $30 \mathrm{~min}$. After centrifugation at $10,000 \mathrm{x} \mathrm{g}$ for $10 \mathrm{~min}$, the supernatant was transferred into a new microtube and the protein concentration was determined by Lowry method at $690 \mathrm{~nm}$. Total protein lysate was freshly used or kept at $-80^{\circ} \mathrm{C}$ until further use.

In-gel digestion. Gel plugs were placed in 96 well-plates and washed with sterile distilled water. The plate was shaken at room temperature for $5 \mathrm{~min}$. The gel plugs were destained at room temperature with $25 \mathrm{mM}$ ammonium bicarbonate $\left(\mathrm{NH}_{4} \mathrm{HCO}_{3}\right)$ in $50 \%$ methanol until they became transparent. Destaining solution was removed and sterile distilled water was added for washing three times. Then the $100 \%$ acetonitrite $(\mathrm{ACN})$, $10 \mathrm{mM}$ DTT in $10 \mathrm{mM} \mathrm{NH}_{4} \mathrm{HCO}_{3}$, was applied to the samples and incubated for $1 \mathrm{~h}$ at room temperature for dehydration and reduction. Alkylation was performed by adding $100 \mathrm{mM}$ iodoacetamide in $10 \mathrm{mM} \mathrm{NH}_{4} \mathrm{HCO}_{3}$ and incubating at room temperature for $1 \mathrm{~h}$ in the dark. Before digestion, the gel slices were washed twice with $100 \% \mathrm{ACN}$ for 5 min. Subsequently, $10 \mathrm{ng} / \mathrm{ml}$ trypsin in $50 \% \mathrm{ACN} / 10 \mathrm{mM} \mathrm{NH} \mathrm{NCO}_{3}$ was added to the gels. The gels were incubated at room temperature for 20 min and kept immersed throughout the digestion with $30 \%$ ACN. Next, the gels were incubated at $37^{\circ} \mathrm{C}$ overnight. To extract the digested peptides, $50 \% \mathrm{ACN}$ in $0.1 \%$ formic acid (FA) was added to the gels and incubated for 10 min whilst shaking. The peptide products were vacuum-dried and stored at $-80^{\circ} \mathrm{C}$ until used for mass spectrometric analysis.

Liquid chromatography-mass spectrometry (LC-MS/MS). Tryptic peptides were analyzed using the HCT ultra PTM Discovery System (Bruker Corporation) and UltiMate 3000 LC System (Thermo Fisher Scientific, Inc.). The peptide samples were dissolved in $0.1 \% \mathrm{FA}$ and the separation was performed 
Table I. Tumor nodule incidence in colons of experimental mice ( 8 mice/group).

\begin{tabular}{clcc}
\hline Group & \multicolumn{1}{c}{ Treatment } & $\begin{array}{c}\text { No. of mice developing } \\
\text { tumor nodules }\end{array}$ & $\begin{array}{c}\text { Incidence of mice } \\
\text { with tumor nodule }(\%)\end{array}$ \\
\hline 1 & Basal diet (negative control) & 0 & 0 \\
2 & Basal diet with AOM/DSS (positive control) & 0 & 87.5 \\
3 & $1.5 \%$ bMO for 5 weeks & 0 & 0 \\
4 & $3.0 \%$ bMO for 5 weeks & 0 & 0 \\
5 & $6.0 \%$ bMO for 5 weeks & 5 & 0 \\
6 & $1.5 \%$ bMO 2 weeks prior to, during, 1 week after AOM/DSS & 3 & 62.5 \\
7 & $3.0 \%$ bMO 2 weeks prior to, during, 1 week after AOM/DSS & 2 & 50.0 \\
8 & $6.0 \%$ bMO 2 weeks prior to, during, 1 week after AOM/DSS & 4 & $37.5^{\mathrm{a}}$ \\
9 & AOM/DSS before feeding with 1.5\% bMO for 14 weeks & 6 & $25.0^{\mathrm{a}}$ \\
10 & AOM/DSS before feeding with 3.0\% bMO for 14 weeks & 50.0 \\
11 & AOM/DSS before feeding with $6.0 \%$ bMO for 14 weeks & 0 & 75.0 \\
12 & $1.5 \%$ bMO for 14 weeks & 0 & 0 \\
13 & $3.0 \%$ bMO for 14 weeks & 0 & 0 \\
14 & $6.0 \%$ bMO for 14 weeks & 0 & 0
\end{tabular}

Differences between tumor incidence in experimental and positive control groups were calculated by the $\chi^{2}$ or Fisher's exact probability test $(16,17)$. ${ }^{\mathrm{a}}<0.05$. bMO, boiled Moringa oleifera pod; AOM, azoxymethane; DSS, dextran sodium sulfate.

on a nanocolumn (Onyx monolithic HDC18; 0.2x150 mm) with electrospray at a flow rate of $300 \mathrm{nl} / \mathrm{min} .0 .1 \% \mathrm{FA}$ and $80 \%$ ACN with $0.1 \%$ FA were used as mobile phase A and mobile phase B, respectively. A multistep gradient was used to elute peptides. The first step involved the increase of B from 10 to $70 \%$ for $13 \mathrm{~min}$, to $90 \% \mathrm{~B}$ at $13-15 \mathrm{~min}$, followed by decreasing to $10 \%$ for $15-20 \mathrm{~min}$. Mass spectra were recorded in the positive-ion mode. A scan range of $300-1,500 \mathrm{~m} / \mathrm{z}$ in AutoMS mode was used for determining the peptide fragment mass spectra. The three averages and five precursor ions were selected from the MS scan 50-3,000 m/z

Protein quantitation and identification. The DeCyder MS 2.0 Differential analysis software (DeCyderMS, GE Healthcare) was used for protein quantitation. The peptide was detected by the PepDetect module and the peptide ion signals were quantified. The Mascot software version 2.2 (Matrix Science, Ltd.) was used for analyzing MS/MS data and protein identification was obtained by searching against NCBI database (https://www.ncbi.nlm.nih.gov). The biological function of each protein was obtained from Uniprot (https://www.uniprot. org) and STITCH 5.0 (http://stitch.embl.de/) databases. A Venn diagram was generated by the jvenn web application (https://xcmsonline.scripps.edu/lib/jvenn-1.8). Protein interaction networks were determined using the STITCH 5.0 database.

Statistical analysis. All data except tumor incidence were expressed as mean \pm SD $(n=8)$. Differences between tumor incidence in experimental and positive control groups were evaluated by the $\chi^{2}$ or Fisher's exact probability test. $\mathrm{P}<0.05$ was considered to indicate a statistically significant difference. All statistical analyses were analyzed by SPSS19.0 software (IBM).

\section{Results}

Effects of bMO on AOM/DSS-induced mouse colon carcinogenesis. The effects of bMO on AOM/DSS-induced CACC were studied in an experimental mouse model, as presented in Fig. 1. Incidence of tumor nodules were examined in 14 experimental groups of mice. No tumor was observed in the negative control (group 1), while the positive control mice (group 2) fed with a basal diet with AOM/DSS demonstrated the highest incidence of tumor nodules $(87.5 \%)$ classified as tubular adenoma (Table I). Group 6-8 mice fed with basal diets containing $1.5,3.0$ and $6.0 \%$ bMO respectively prior to induction with AOM/DSS had lower incidences of tumor nodules compared with the positive control mice (Table I). Notably, mice fed with 6.0\% bMO diet (group 8) had a significantly lower tumor nodule incidence compared with the positive control mice $(37.5 \%, \mathrm{P}<0.05)$, while tumor nodules were not observed in group 3-5 mice fed with 1.5, 3.0 and $6.0 \%$ bMO diets without AOM/DSS induction, respectively (Table I). In addition, group 9-11 mice treated with AOM/DSS and subsequently fed with 1.5, 3 and 6.0\% bMO diets, respectively from week 6 until the end of the experiment also demonstrated lower incidences of tumor nodules compared with positive control mice (group 2). In particular, mice fed with a $1.5 \%$-bMO diet (group 9) had a significantly lower tumor nodule incidence $(25.0 \%)$ compared with the positive control mice $(\mathrm{P}<0.05$; Table $\mathrm{I})$. No tumor nodule was observed in group 12-14 mice fed with the same feeding regimen without AOM/DSS induction (Table I). Based on the significant reduction of tumor incidence observed in mice receiving $6.0 \%$ bMO prior to induction with AOM/DSS, designated as the preventive consumption, and $1.5 \% \mathrm{bMO}$ diets after inducing with AOM/DSS, assigned as therapeutic consumption, colon samples from 6 experimental groups 
(group 1, 2, 5, 8, 9 and 12) were chosen for further comparative analysis of protein expression profiles expressed in response to bMO consumption in the mouse model of CACC.

Protein expression profiles associated with the effects of bMO on AOM/DSS-induced mouse colon carcinogenesis. To explore the protein expression profiles that underlie the effects of bMO consumption on AOM/DSS-induced mouse colon carcinogenesis, total proteins were extracted from the colons of mice and subjected to analysis using GeLC-MS/MS. The analysis demonstrated a total of 125 proteins that were differentially expressed in tissue samples from all 14 groups of mice. Functional annotations of the proteins, obtained from online Uniprot and STITCH 5.0 databases, are presented in Fig. 2A. These proteins were associated with various biological processes, including signal transduction (13.6\%), metabolism $(12.0 \%)$, cytoskeleton (11.2\%), transcription (11.2\%), translation (10.4\%), cellular development (6.4\%), transport (4.8\%), protein modification (4.8\%), stress response (4.0\%), cell division $(2.4 \%)$, cell adhesion $(2.4 \%)$, immune response $(1.6 \%)$, DNA repair $(1.6 \%)$, meiosis $(0.8 \%)$, hemostasis $(0.8 \%)$, energy $(0.8 \%)$, chaperone pathway $(0.8 \%)$, sarcomere organization $(0.8 \%)$, chromatin remodeling $(0.8 \%)$ and unknown $(8.8 \%)$.

A Venn diagram was used to demonstrate the numbers of proteins differentially expressed in 6 selected experimental groups of mice designed to assess preventive and therapeutic effects of bMO, as presented in Fig. 2B. There were 99 proteins identified in the negative control group, 119 proteins in AOM/DSS-treated mice, 112 proteins in mice supplemented with $1.5 \%$ bMO, 115 proteins in the $\mathrm{AOM} / \mathrm{DSS}$-induced mice receiving therapeutic $1.5 \% \mathrm{bMO}$ diets, 117 proteins in mice supplemented with $6.0 \%$ bMO and 113 proteins in the AOM/DSS-induced mice receiving preventive $6.0 \%$ bMO diets. There were seven proteins potentially associated with the preventive and therapeutic effects of bMO on AOM/DSS-induced CACC, as indicated by red arrows in Fig. 2B. These proteins were classified into three groups, based on their expression patterns described in Table II. A total of three proteins in group 1, 60S acidic ribosomal protein $\mathrm{P} 1$, fragile $\mathrm{X}$ mental retardation 1 (FMR1) and cystatin 9, were detectable in control mice and downregulated in AOM/DSS-induced mice. Notably, all three proteins were upregulated in response to both preventive and therapeutic supplementation with bMO. In contrast, two proteins (group 2), STDP2 and zinc finger protein 638 , were undetectable in the control group and were expressed in response to the AOM/DSS induction of CACC. STDP2 appeared to be undetectable in all mice treated with preventive and therapeutic bMO, while zinc finger protein 638 proteins were not found in preventive bMO-treated and control therapeutic $1.5 \%$ bMO groups and remained detectable in therapeutic $1.5 \%$ bMO-supplemented mice. Additionally, magnesium-dependent protein phosphatase $2 \mathrm{C}$ and unnamed protein product (group 3) were undetectable in control and AOM/DSS-induced mice. These two proteins were upregulated in response to both preventive and therapeutic bMO supplementation, however unnamed protein product was undetectable in the AOM/DSS induced mice fed with therapeutic $1.5 \% \mathrm{bMO}$. These data indicated a potential association of these seven proteins in the preventive and therapeutic effects of $\mathrm{bMO}$ on AOM/DSS-induced mouse CACC.
STITCH analysis for protein-protein interaction networks of candidate proteins. In order to identify the molecular mechanisms underlying the preventive and therapeutic effects of $\mathrm{bMO}$ on AOM/DSS-induced carcinogenesis, protein-protein interaction networks of the seven proteins obtained from Venn diagram analysis were analyzed. Using the online STITCH 5.0 resource, the networks were predicted with parameters limited to Mus musculus, medium confidence score (0.400) and no more than 10 interactors. All the candidate proteins except for FMR1, round spermatids protein STDP2 and unnamed protein product were found in the analysis. The analysis included ribosomal protein large P1 (Rplp1), cystatin 9 (Cst9), zinc finger protein 638 or zinc finger matrin-like protein (ZfmL), and magnesium dependent protein phosphatase $1 \mathrm{G}$ (formerly 2C) (Ppm1g). As our previous studies indicated that bMO has anticancer properties, as it induces apoptosis and is anti-inflammatory $(15,17)$, the associations of these candidate proteins with apoptotic and inflammatory signaling pathways were determined. The protein-protein interaction with key regulators of apoptosis, BCL2-associated X protein (Bax), B cell leukemia/lymphoma 2 (BCL-2) and caspases (Casp) were analyzed (Fig. 3A). The data demonstrated that only Rplp1 protein was associated with this apoptotic signaling network, whereas the other proteins, Cst9, $\mathrm{Zfml}$ and Ppmlg, did not show any associations (Fig. 3A). Analysis of the interaction between the aforementioned proteins and important inflammatory molecules, tumor necrosis factor- $\alpha$ (TNF- $\alpha$ ), Fas ligand (Fasl), inducible nitric oxide synthase (NOS2), endothelial nitric oxide synthase (NOS3) and interleukin-6 (IL-6), signal transducer and activator of transcription 3 (STAT3) is presented in Fig. 3B. The data indicated that Rplp1 and Ppm1g were involved in inflammatory pathways, while Cst 9 and $\mathrm{Zfml}$ proteins did not demonstrate any interaction. The STITCH analysis indicated that of a total of seven candidate proteins potentially associated with the preventive and therapeutic effects of bMO in AOM/DSS-induced carcinogenesis, Rplp1 and Ppmlg proteins had potential interactions with apoptotic and inflammatory signaling pathways.

\section{Discussion}

Previous studies in an experimental mouse model have demonstrated the preventive and therapeutic effects of bMO on the AOM/DSS-induced CACC $(16,17)$. In the present study investigating the effects of $1.5,3.0$ and $6.0 \%$ bMO consumption on colon tumor incidence, a significant preventive effect of bMO was observed only in mice receiving $6.0 \%$ bMO diets, while mice fed with therapeutic diets containing $1.5 \%$ bMO demonstrated a significant decrease in the number of tumor nodules. Basically, vitamins, minerals and dietary phytochemicals exhibited both antioxidant and prooxidant activities. The lower efficacy of high-dose bMO in the present study was possibly due to the activity of bioactive compounds in MO pods including glucosinolates, isothiocyanates and $\beta$-carotene (19). These results are consistent with those of a previous study in AOM-induced rats. Rats that received 100 or $200 \mathrm{ppm}$ of $\beta$-carotene inhibited the aberrant cryptic foci (ACF) formation, whereas administration of higher doses, 1,000 or $2,000 \mathrm{ppm}$ of $\beta$-carotene increased colonic ACF formation (20). 

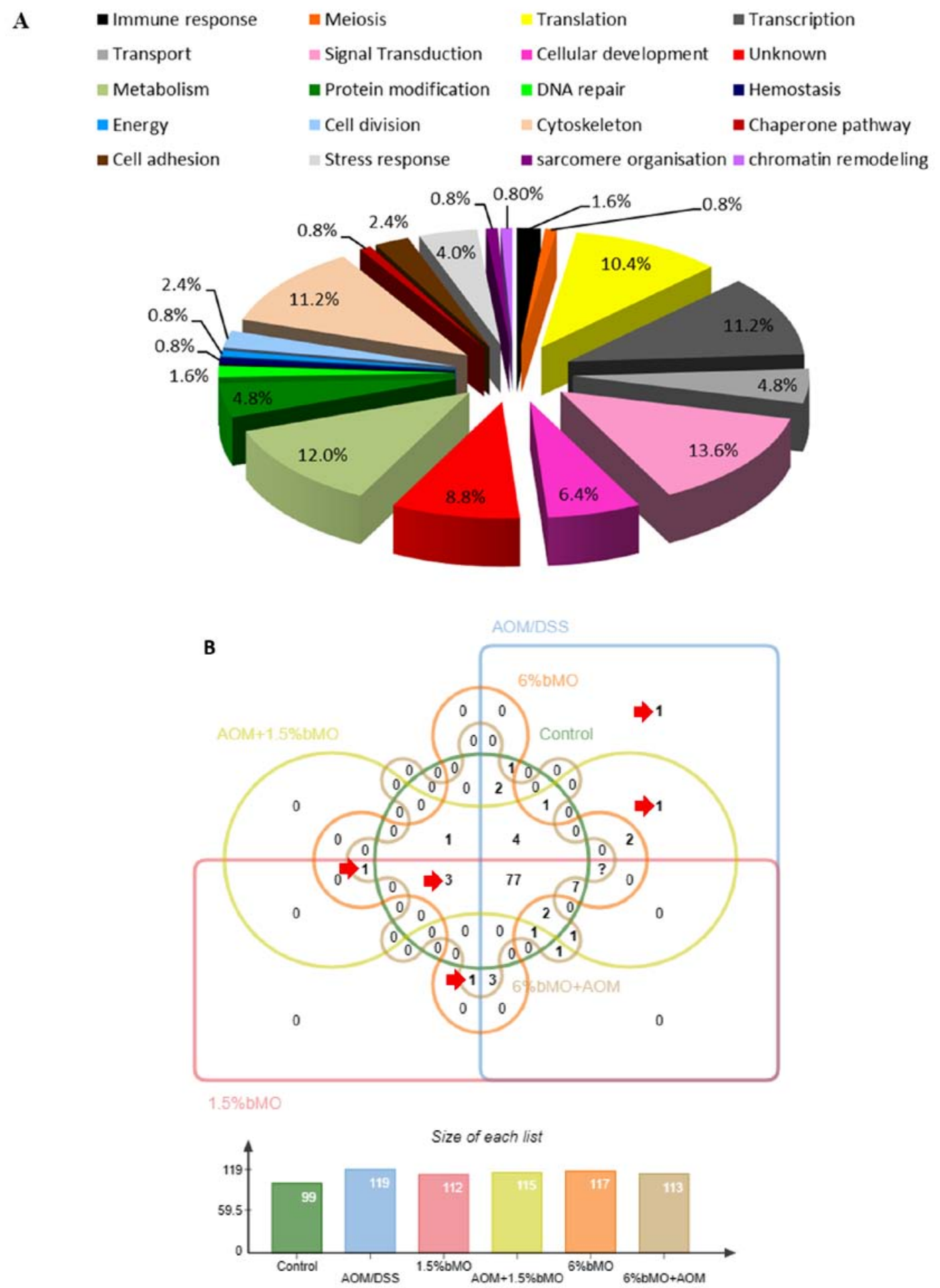

Figure 2. (A) Functional annotation of 125 proteins differentially expressed in the 14 groups of experimental mice. Data were obtained from the online Uniprot (http://www.uniprot.org) and STITCH 5.0 databases (http://stitch.embl.de/). (B) Venn diagram presenting numbers of proteins expressed differentially in 6 selected groups of mice: Negative control, positive control, mice receiving 1.5\% bMO diet, mice receiving 1.5\% bMO diet followed by the AOM/DSS induction, mice fed with $6.0 \% \mathrm{bMO}$ and mice fed with $6.0 \%$ diet before the AOM/DSS induction. X-axis represents different experimental groups of mice while Y-axis represents the number of proteins found in each group. Red arrows indicate the seven candidate proteins potentially associated with the preventive and therapeutic effects of bMO. A question mark represents 15 proteins expressed in the indicated groups. bMO, boiled Moringa oleifera pod; AOM, azoxymethane; DSS, dextran sodium sulfate.

In the present study, the comparative analysis of protein expression using the GeLC-MS/MS technique in mouse colon tissues from all 14 groups of mice revealed that 125 proteins were differentially expressed. These proteins were identified to be involved in various biological activities, including signal transduction, metabolism, cytoskeleton, transcription, translation, cellular development, transport, protein modification, stress response, cell division, cell adhesion, immune 


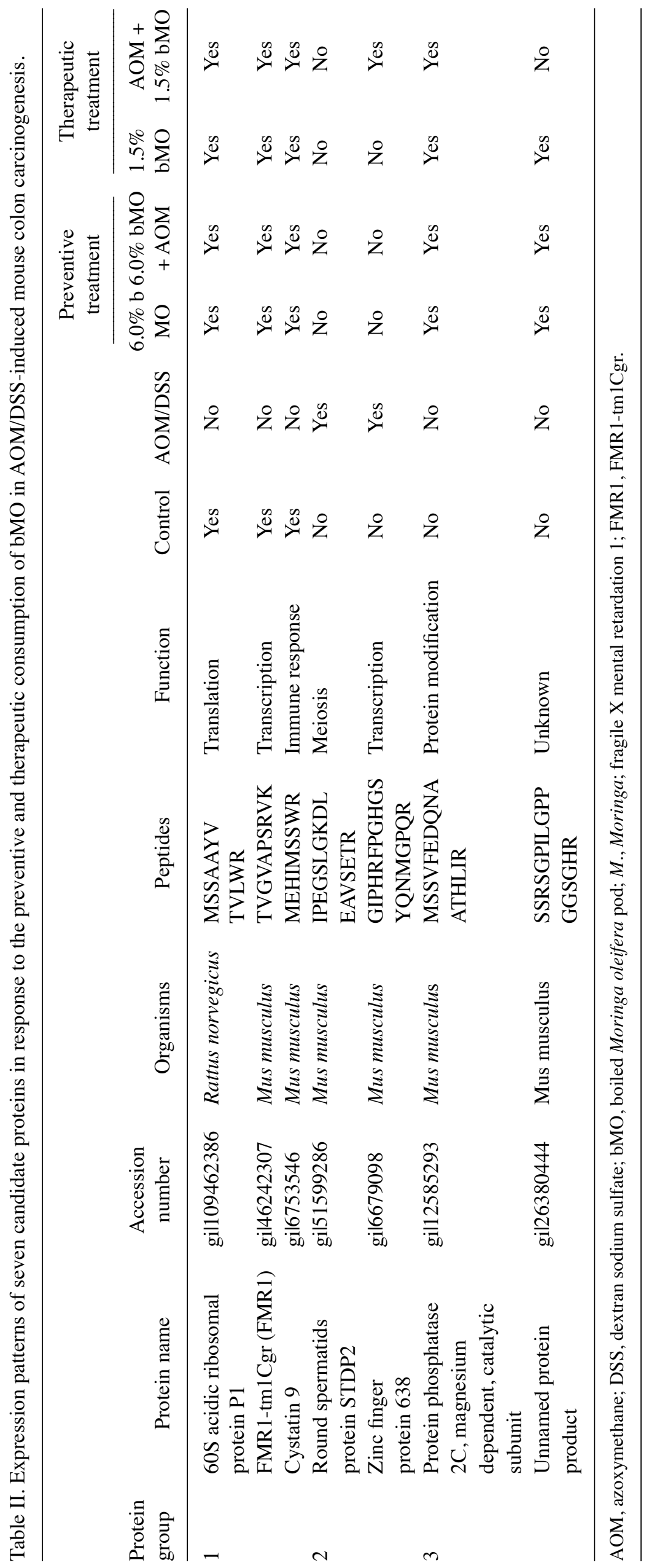


A
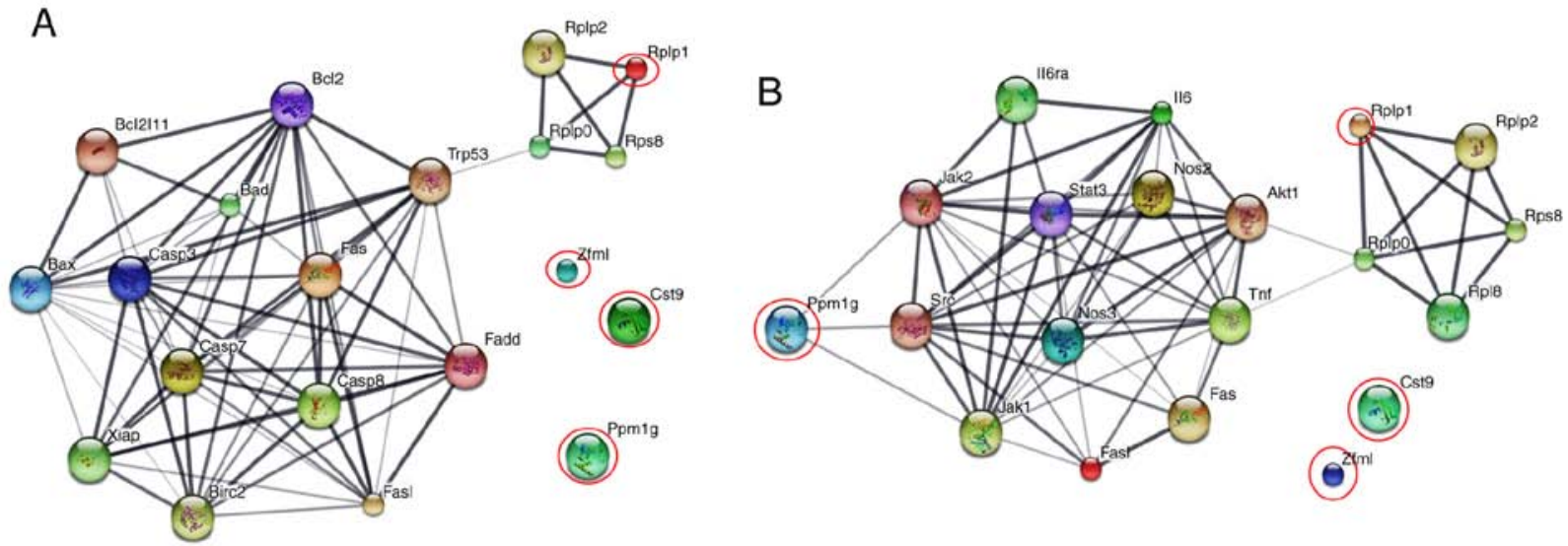

Figure 3. Protein interaction networks of candidate proteins and their association with apoptotic and inflammatory signaling pathways. (A) and (B) present the associations of candidate proteins with apoptotic and inflammatory signaling networks, respectively. Red circles indicate the input proteins identified in this study. Edges represent different levels of meaningful protein-protein associations. Stronger associations are presented by thicker lines and weaker associations by thinner lines. Bax, Bcl-2-associated X protein; Bcl2 (Bcl-2), B-cell lymphoma 2; casp3, caspase 3; casp7, caspase 7; TNF, tumor necrosis factor; NOS2, inducible nitric oxide synthase; NOS3, endothelial nitric oxide synthase; IL-6, interleukin-6; STAT 3, signal transducer and activator of transcription 3.

response, DNA repair, meiosis, hemostasis, energy, the chaperone pathway, sarcomere organization and chromatin remodeling. Notably, there were seven candidate proteins found that were potentially expressed in response to bMO consumption in AOM/DSS-induced CACC and serve key roles in the suppression associated with bMO. Using the online STITCH 5.0 database, all the candidate proteins were found except for FMR1, STDP2 and unnamed protein product and the association of Rplp1 and Ppm1g proteins with inflammatory and apoptotic signaling networks were demonstrated. The data obtained in the present study is consistent with previous reports demonstrating that preventive and therapeutic efficacies of bMO were partially based on the induction of apoptosis in colon tissues and reduction of proinflammatory cytokines and mediators in the mouse CACC model (15-17).

In the present study, ribosomal protein $\mathrm{P} 1$ expression was downregulated in mouse AOM/DSS-induced CACC, and was detectable in mice receiving both preventive and therapeutic bMO supplementation, indicating their potential roles in mouse $\mathrm{CACC}$, and the preventive and therapeutic potentials of bMO in colon cancer. Rplp1 is a member of the acidic ribosomal proteins (RPs) found in the ribosomal stalk $(21,22)$. The stalk is known as a pentameric $\mathrm{P}$ protein complex comprising RP large P0 (Rplp0) and two heterodimers formed by RP large P1 (Rplp1) and RP large P2 (Rplp2), which play important roles in the translation process $(21,22)$. Alterations of Rplp protein expression have been reported in human cancer, such as colorectal and pancreatic cancer $(23,24)$. In contrast to the findings of the present study that demonstrated the downregulation of Rplp1 expression in mice with CACC compared with normal mouse control, a previous study indicated a significant increase of Rplp0, Rplp1 and Rplp2 protein expression in breast, skin, colon, lung and ovarian tumors with respect to corresponding normal tissues and the presence of metastasis in gynecological cancer (25). Furthermore, co-expression of Rplp1 with mutant rasVal12 caused the transformation of murine NIH3T3 cells (26). However, Rplp1 was demonstrated to be essential for cell proliferation, mouse embryonic and brain development (27). Downregulation of Rplp0, Rplp1 and Rplp2 proteins led to reactive oxygen species (ROS) accumulation, endoplasmic reticulum (ER) stress/unfolded protein response (UPR) activation, and induced autophagy, and inhibition of ROS production resulted in restoring of proliferative capacity in breast and ovarian cancer cells (28). The STITCH analysis performed in the present study demonstrated relationships between Rplp proteins and apoptotic and inflammatory signaling networks. This data is consistent with previous studies demonstrating that there is a link between the expression of Rplp1 and the tumor suppressor gene p53 in vitro and in vivo (26-28), and that a deficiency of Rplp resulted in the reduction of mTOR and Akt phosphorylation in MCF-7 breast cancer cells (28). To the best of our knowledge at present there is no direct evidence indicating a functional role of Rplp1 in apoptosis and inflammation involving colorectal carcinogenesis. While precise biological and physiological functions of Rplp remain largely unclear, the downregulation of Rplp1 expression observed in the present study suggested that Rplp1 has a protective role in AOM/DSS-induced mouse CACC and Rplp1 is probably one of the preventive and therapeutic targets of bMO supplementation.

Similarly, FMR1 and Cst9 proteins were downregulated in mouse AOM/DSS-induced CACC and appeared to be detectable in mice receiving both preventive and therapeutic bMO supplementation. FMR1 proteins are encoded by the FMR1 gene whose inactivation causes fragile $X$ syndrome (FXS) characterized phenotypically and molecularly in a knockout mouse model (29). The fragile $\mathrm{X}$ mental retardation protein (FMRP), an RNA-binding protein that serves roles in RNA metabolism (30), has been demonstrated directly or indirectly to influence cancer progression. Previous studies indicated overexpression of FMR1 mRNA in hepatocellular carcinoma cells $(31,32)$ and patients with FXS had a decreased risk of cancer (33). The FMRP gene also modulated several malignant phenotypes required for the metastasis and invasion of melanoma and breast cancer $(34,35)$.

Cystatin 9 (Cst9) proteins, also known as testatin, are characterized as a member of the cysteine protease inhibitors named 
Cystatin family, and are expressed in a restricted manner in germ cells and somatic cells in reproductive tissues (36). A few studies have indicated that $\mathrm{Cst} 9$ proteins are involved in testis development and have immunomodulatory and antimicrobial functions (36-38). Although the functional properties of Cst9 remain unclear, accumulating evidence implicates cystatins and their target lysosomal proteases, termed the cathepsins, in tumorigenesis and metastasis of various cancer types, such as brain tumors $(39,40)$. The cystatins appear to exert their tumor suppressor activity by counter-balancing the overexpressed tumor-associated proteolytic activity of the cathepsins $(39,40)$. In the present study, Cst9, but not FMR1, was found in the STITCH 5.0 database and was not found to be associated with apoptosis and inflammatory signaling pathways. At present, there is a lack of direct evidence supporting the roles of FMR1 and Cst9 proteins in colorectal carcinogenesis, and the results of the proteomic analysis performed in the present study may suggest the potential implication of FMR1 and Cst9 in mouse AOM/DSS-induced carcinogenesis and response to preventive and therapeutic bMO treatments.

The proteomic analysis performed in the present study found that STDP2 and ZNF638 were expressed in response to AOM/DSS treatment in AOM/DSS-induced mouse CACC. The STDP2 protein is encoded by identified novel genes with evident testis-specific expression, characterized by in silico and in vitro approaches in spermatogenetic cells (41). Presently, there is a lack of evidence demonstrating the precise function of STDP2, consistent with an absence of STDP2 in STITCH resources. ZNF638 alternatively named as zinc finger matrin-like ( $\mathrm{Zfml}$ ), is one of the zinc-finger domain containing proteins participating in a wide range of molecular and cellular activities (42). While there are several other zinc-finger proteins such as ZNF281, ZNF750 and ZNF185, having oncogenic and tumor suppressor functions, the ZNF638 protein has been reported as an early regulator of adipogenesis (42). The protein appears to act as a transcriptional cofactor of CCAAT/enhancer-binding protein, leading to the expression of peroxisome proliferator-activated receptor $\gamma$, the key regulator of adipocyte differentiation (43). The STITCH analysis performed in the present study indicated that there was no association between ZNF638 protein and apoptosis or inflammation. In the present study, while both preventive and therapeutic bMO consumption were able to downregulate STDP2 expression, ZNF638 was downregulated in response to only the preventive supplementation. To the best of our knowledge, the present proteomic study is the first report suggesting the potential involvement of STDP2 and ZNF638 in mouse $\mathrm{CACC}$ and the suppressive potentials of bMO in colorectal cancer.

In the experimental mouse model used in the present study, Ppm1g and unnamed protein product (gil26380444) were not involved in the AOM/DSS-induced mouse CACC. They were likely to be upregulated in response to both preventive and therapeutic bMO consumption, although expression of unnamed protein product was not observed in the AOM/DSS-induced mice fed with therapeutic bMO diet. Whereas there is no information of the unnamed protein product, $\mathrm{Ppm} 1 \mathrm{~g}$ is well-characterized. Ppm1g is a member of Type 2C phosphatase family (PP2C or PPM) of Ser/Thr protein phosphatases that possess unique patterns of tissue and subcellular distribution associated with diverse functionalities (44). PP2C isoforms have been implicated in signaling networks controlling cell differentiation, proliferation, growth, survival and metabolism (44). Previous studies indicated that the dephosphorylation of a cyclin-CDK inhibitor p27 by $\mathrm{Ppm} 1 \mathrm{~g}$ maintained the proper level of the $\mathrm{p} 27$ proteins that basically induce cell cycle arrest at G1 phase to prevent the improper entry to cell cycle (45). Ppm1g has also been demonstrated to participate in the DNA damage signal leading to tumor suppressor p53-dependent response (46). In the present study, STITCH analysis suggested the association of Ppm1g protein with inflammatory, but not with apoptotic signaling networks. These results are contrary to previous studies that demonstrated the anti-apoptotic activity of Ppm1g in mouse neural development (47) and found that Ppm1g is a transcriptional coactivator of $\mathrm{NF}-\kappa \mathrm{B}$ in the tumor necrosis factor- $\alpha$-mediated inflammatory pathways (48). Therefore, the upregulation of Ppm1g in response to the preventive and therapeutic supplementation with bMO observed in the present study may explain the potential of bMO in this mouse colon carcinogenesis model.

The data generated in the present study provide insight into the molecular mechanisms of the suppressive potential of MO consumption in mouse colon carcinogenesis, based on the expression of the seven candidate proteins in response to MO in colon tissues. Nevertheless, further in vitro and in vivo studies are required to investigate expression and function of the candidate proteins in colon cancers and the lack of experimental validation of protein expression may be noted as a limitation of the present study. The proteomic findings of this study support the potential of MO-supplemented diets in cancer treatment and care.

\section{Acknowledgements}

The authors would like to thank Associate Professor Dr Anudep Rangsripipat from the Faculty of Veterinary Science, Chulalongkorn University, Thailand for his assistance with the pathological examination of mouse tissue. The authors would also like to thank Professor Jeong-Sang Lee from Jeonju University, Korea for his helpful guidance on constructing the AOM/DSS-induced mouse colon carcinogenesis model.

\section{Funding}

This work was supported by grants from the National Center for Genetic Engineering and Biotechnology (grant no. P-09-00762), National Science and Technology Development Agency and Ministry of Science and Technology, Thailand.

\section{Availability of data and materials}

The datasets used and/or analyzed during the current study are available from the corresponding author on reasonable request.

\section{Authors' contributions}

PP performed data analysis and drafted the manuscript. PK, SB and $\mathrm{CB}$ performed the animal experiments and data analysis. 
SR and NP contributed to proteomic analysis. ST designed the experiments and edited the manuscript for important intellectual content. CA conducted proteomic analysis, interpreted data and wrote the manuscript. All authors have read and approved the manuscript.

\section{Ethics approval and consent to participate}

The present study was approved by the Animal Ethics Committee of the National Cancer Institute (approval no. ACUC 2009/003) and Animal Care and Use Protocol of Mahidol University (Nakhon Pathom, Thailand).

\section{Patient consent for publication}

Not applicable.

\section{Competing interests}

The authors declare that they have no competing interests.

\section{References}

1. Arnold M, Sierra MS, Laversanne M, Soerjomataram I, Jemal A and Bray F: Global patterns and trends in colorectal cancer incidence and mortality. Gut 66: 683-691, 2017.

2. Dolatkhah R, Somi MH, Kermani IA, Ghojazadeh M, Jafarabadi MA, Farassati F and Dastgiri S: Increased colorectal cancer incidence in Iran: A systematic review and meta-analysis. BMC Public Health 15: 997, 2015.

3. Yin TF, Wang M, Qing Y, Lin YM and Wu D: Research progress on chemopreventive effects of phytochemicals on colorectal cancer and their mechanisms. World J Gastroenterol 22: 7058-7068, 2016.

4. Bradbury KE, Appleby PN and Key TJ: Fruit, vegetable, and fiber intake in relation to cancer risk: Findings from the European prospective investigation into cancer and nutrition (EPIC). Am J Clin Nutr 100 (Suppl 1): S394-S398, 2014.

5. Abdull Razis AF, Ibrahim MD and Kntayya SB: Health benefits of moringa oleifera. Asian Pac J Cancer Prev 15: 8571-8576, 2014.

6. Karim NA, Ibrahim MD, Kntayya SB, Rukayadi Y, Hamid HA and Razis AFA: Moringa oleifera Lam: Targeting chemoprevention. Asian Pac J Cancer Prev 17: 3675-3686, 2016.

7. Leone A, Spada A, Battezzati A, Schiraldi A, Aristil J and Bertoli S: Cultivation, genetic, ethnopharmacology, phytochemistry and pharmacology of Moringa oleifera leaves: An overview. Int J Mol Sci 16: 12791-12835, 2015.

8. Francis JA, Jayaprakasam B, Olson LK and Nair MG: Insulin secretagogues from Moringa oleifera with cyclooxygenase enzyme and lipid peroxidation inhibitory activities. Helv Chim Acta 87: 317-326, 2004.

9. Cheenpracha S, Park EJ, Yoshida WY, Barit C, Wall M, Pezzuto JM and Chang LC: Potential anti-inflammatory phenolic glycosides from the medicinal plant Moringa oleifera fruits. Bioorg Med Chem 18: 6598-6602, 2010.

10. Al-Asmari AK, Albalawi SM, Athar MT, Khan AQ, Al-Shahrani $\mathrm{H}$ and Islam $\mathrm{M}$ : Moringa oleifera as an anti-cancer agent against breast and colorectal cancer cell lines. PLoS One 10: e0135814, 2015.

11. Elsayed EA, Sharaf-Eldin MA and Wadaan M: In vitro evaluation of cytotoxic activities of essential oil from moringa oleifera seeds on HeLa, HepG2, MCF-7, CACO-2 and L929 cell lines. Asian Pac J Cancer Prev 16: 4671-4675, 2015.

12. Suphachai C: Antioxidant and anticancer activities of Moringa oleifera leaves. J Med Plants Res 8: 318-325, 2014.

13. Potestà M, Minutolo A, Gismondi A, Canuti L, Kenzo M, Roglia V, Macchi F, Grelli S, Canini A, Colizzi V and Montesano C: Cytotoxic and apoptotic effects of different extracts of Moringa oleifera Lam on lymphoid and monocytoid cells. Exp Ther Med 18: 5-17, 2019.
14. Pirrò S, Matic I, Guidi A, Zanella L, Gismondi A, Cicconi R, Bernardini R, Colizzi V, Canini A, Mattei M and Galgani A: Identification of microRNAs and relative target genes in Moringa oleifera leaf and callus. Sci Rep 9: 15145, 2019.

15. Muangnoi C, Chingsuwanrote P, Praengamthanachoti P, Svasti $S$ and Tuntipopipat S: Moringa oleifera pod inhibits inflammatory mediator production by lipopolysaccharide-stimulated RAW 264.7 murine macrophage cell lines. Inflammation 35: 445-455, 2012.

16. Budda S, Butryee C, Tuntipopipat S, Rungsipipat A, Wangnaithum S, Lee JS and Kupradinun P: Suppressive effects of Moringa oleifera Lam pod against mouse colon carcinogenesis induced by azoxymethane and dextran sodium sulfate. Asian Pac J Cancer Prev 12: 3221-3228, 2011.

17. Kraiphet S, Butryee C, Rungsipipat A, Budda S, Rattanapinyopitak K and Tuntipopipat S: Apoptosis induced by Moringa oleifera Lam. pod in mouse colon carcinoma model. Comp Clin Pathol 27: 21-30, 2018.

18. Kosulwat V, Rojroongwasinkul N, Boonpraderm A, Viriyapanich T, Jitnarin N, Sornkaew N and Vanicchakul C: Food consumption data of Thailand. Bangkok: National Bureau of Agricultural Commodity and Food Standards, Ministry of Agriculture and Cooperatives: Bangkok, Thailand, 2006 (In Thai).

19. Fahey JW: Moringa oleifera: A review of the medical evidence for its nutritional, therapeutic, and prophylactic properties. Part 1. Trees Life J 1: 5, 2005.

20. Raju J, Swamy MV, Cooma I, Patlolla JM, Pittman B, Reddy BS, Steele VE and Rao CV: Low doses of beta-carotene and lutein inhibit AOM-induced rat colonic ACF formation but high doses augment ACF incidence. Int J Cancer 113: 798-802, 2005.

21. Naganuma T, Shiogama K and Uchiumi T: The N-terminal regions of eukaryotic acidic phosphoproteins $\mathrm{P} 1$ and $\mathrm{P} 2$ are crucial for heterodimerization and assembly into the ribosomal GTPase-associated center. Genes Cells 12: 501-510, 2007.

22. Xu X, Xiong X and Sun Y: The role of ribosomal proteins in the regulation of cell proliferation, tumorigenesis, and genomic integrity. Sci China Life Sci 59: 656-672, 2016

23. Zhang L, Zhou W, Velculescu VE, Kern SE, Hruban RH, Hamilton SR, Vogelstein B and Kinzler KW: Gene expression profiles in normal and cancer cells. Science 276: 1268-1272, 1997.

24. Loging WT and Reisman D: Elevated expression of ribosomal protein genes L37, RPP-1, and S2 in the presence of mutant p53. Cancer Epidemiol Biomarkers Prev 8: 1011-1016, 1999.

25. Artero-Castro A, Castellvi J, García A, Hernández J, Ramón y Cajal S and Lleonart ME: Expression of the ribosomal proteins Rplp0, Rplp1, and Rplp2 in gynecologic tumors. Hum Pathol 42: 194-203, 2011.

26. Artero-Castro A, Kondoh H, Fernández-Marcos PJ, Serrano M, Ramón y Cajal S and Lleonart ME: Rplp1 bypasses replicative senescence and contributes to transformation. Exp Cell Res 315: 1372-1383, 2009

27. Perucho L, Artero-Castro A, Guerrero S, Ramón y Cajal S, LLeonart ME and Wang ZQ: RPLP1, a crucial ribosomal protein for embryonic development of the nervous system. PLoS One 9: e99956, 2014

28. Artero-Castro A,Perez-Alea M,Feliciano A,Leal JA, Genestar M, Castellvi J, Peg V, Ramón Y Cajal S and Lleonart ME: Disruption of the ribosomal $\mathrm{P}$ complex leads to stress-induced autophagy. Autophagy 11: 1499-1519, 2015.

29. Yan QJ, Asafo-Adjei PK, Arnold HM, Brown RE and Bauchwitz RP: A phenotypic and molecular characterization of the fmr1-tm1Cgr fragile X mouse. Genes Brain Behav 3: 337-359, 2004.

30. Bagni C, Tassone F, Neri G and Hagerman R: Fragile X syndrome: Causes, diagnosis, mechanisms, and therapeutics. J Clin Invest 122: 4314-4322, 2012.

31. Li Y, Tang Y, Ye L, Liu B, Liu K, Chen J and Xue Q: Establishment of a hepatocellular carcinoma cell line with unique metastatic characteristics through in vivo selection and screening for metastasis-related genes through cDNA microarray. J Cancer Res Clin Oncol 129: 43-51, 2003.

32. Liu Y, Zhu X, Zhu J, Liao S, Tang Q, Liu K, Guan X, Zhang J and Feng Z: Identification of differential expression of genes in hepatocellular carcinoma by suppression subtractive hybridization combined cDNA microarray. Oncol Rep 18: 943-951, 2007.

33. Schultz-Pedersen S, Hasle H, Olsen JH and Friedrich U: Evidence of decreased risk of cancer in individuals with fragile X. Am J Med Genet 103: 226-230, 2001. 
34. Lucá R, Averna M, Zalfa F, Vecchi M, Bianchi F, La Fata G, Del Nonno F, Nardacci R, Bianchi M, Nuciforo P, et al: The fragile $\mathrm{X}$ protein binds mRNAs involved in cancer progression and modulates metastasis formation. EMBO Mol Med 5: 1523-1536, 2013.

35. Zalfa F,Panasiti V,Carotti S,Zingariello M,Perrone G, Sancillo L, Pacini L, Luciani F, Roberti V, D'Amico S, et al: The fragile X mental retardation protein regulates tumor invasiveness-related pathways in melanoma cells. Cell Death Dis 8: e3169, 2017.

36. Töhönen V, Osterlund $C$ and Nordqvist K: Testatin: A cystatin-related gene expressed during early testis development. Proc Natl Acad Sci USA 95: 14208-14213, 1998.

37. Hasegawa K, Chuma S, Tada T, Sakurai T, Tamura M, Suemori H and Nakatsuji N: Testatin transgenic and knockout mice exhibit normal sex-differentiation. Biochem Biophys Res Commun 341: 369-375, 2006

38. Eaves-Pyles T, Patel J, Arigi E, Cong Y, Cao A, Garg N, Dhiman M, Pyles RB, Arulanandam B, Miller AL, et al: Immunomodulatory and antibacterial effects of cystatin 9 against Francisella tularensis. Mol Med 19: 263-275, 2013.

39. Magister S and Kos J: Cystatins in immune system. J Cancer 4: 45-56, 2013.

40. Strojnik T, Zajc I, Bervar A, Zidanik B, Golouh R, Kos J, Dolenc V and Lah T: Cathepsin B and its inhibitor stefin A in brain tumors. Pflugers Arch 439 (3 Suppl): R122S-R123S, 2000.

41. Hong S, Choi I, Woo JM, Oh J, Kim T, Choi E, Kim TW, Jung YK, $\mathrm{Kim} \mathrm{DH}$, Sun $\mathrm{CH}$, et al: Identification and integrative analysis of 28 novel genes specifically expressed and developmentally regulated in murine spermatogenic cells. J Biol Chem 280: 7685-7693, 2005.
42. Cassandri M, Smirnov A, Novelli F, Pitolli C, Agostini M, Malewicz M, Melino G and Raschellà G: Zinc-finger proteins in health and disease. Cell Death Discov 3: 17071, 2017.

43. Meruvu S, Hugendubler L and Mueller E: Regulation of adipocyte differentiation by the zinc finger protein ZNF638. J Biol Chem 286: 26516-26523, 2011.

44. Lu G and Wang Y: Functional diversity of mammalian type $2 \mathrm{C}$ protein phosphatase isoforms: New tales from an old family. Clin Exp Pharmacol Physiol 35: 107-112, 2008.

45. Sun C, Wang G, Wrighton KH, Lin H, Songyang Z, Feng XH and Lin X: Regulation of $\mathrm{p} 27^{\mathrm{Kipl}}$ phosphorylation and $\mathrm{G} 1$ cell cycle progression by protein phosphatase PPM1G. Am J Cancer Res 6: 2207-2220, 2016.

46. Khoronenkova SV, Dianova II, Ternette N, Kessler BM, Parsons JL and Dianov GL: ATM-dependent downregulation of USP7/HAUSP by PPM1G activates p53 response to DNA damage. Mol Cell 45: 801-813, 2012.

47. Foster WH, Langenbacher A, Gao C, Chen J and Wang Y: Nuclear phosphatase PPM1G in cellular survival and neural development. Dev Dyn 242: 1101-1109, 2013.

48. McNamara RP, McCann JL, Gudipaty SA and D'Orso I: Transcription factors mediate the enzymatic disassembly of promoter-bound 7SK snRNP to locally recruit P-TEFb for transcription elongation. Cell Rep 5: 1256-1268, 2013.

This work is licensed under a Creative Commons Attribution-NonCommercial-NoDerivatives 4.0 International (CC BY-NC-ND 4.0) License. 